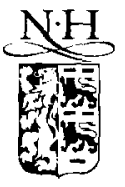

ELSEVIER
13 October 1994

PHYSICS LETTERS B

Physics Letters B 337 (1994) 376-382

\title{
Study of the primary cosmic ray composition around the knee of the energy spectrum
}

\section{EAS-TOP Collaboration}

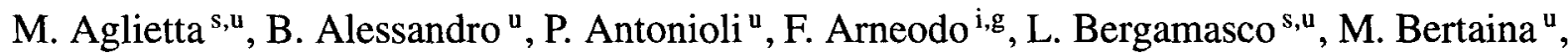

A. Campos Fauth ${ }^{\mathrm{v}}$, C. Castagnoli ${ }^{\mathrm{s}, \mathrm{u}}, \mathrm{A}$. Castellina ${ }^{\mathrm{s}, \mathrm{u}}$, C. Cattadori ${ }^{\mathrm{t}}, \mathrm{A}$. Chiavassa ${ }^{\mathrm{s}, \mathrm{u}}$, G. Cini ${ }^{s, u}$, B. D'Ettorre Piazzoli ${ }^{\ell}$, G. Di Sciascio ${ }^{\ell}$, W. Fulgione ${ }^{s, u}$, P. Galeotti ${ }^{\text {s,u }}$, P.L. Ghia ${ }^{\text {s,u }}$,

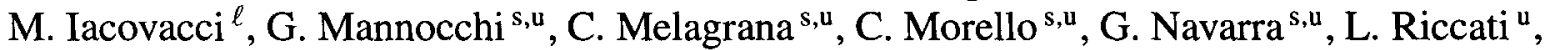
O. Saavedra ${ }^{\mathrm{s}, \mathrm{u}}$, G.C. Trinchero ${ }^{\mathrm{s}, \mathrm{u}}$, P. Vallania ${ }^{\mathrm{s}, \mathrm{u}}, \mathrm{S}$. Vernetto ${ }^{\mathrm{s}, \mathrm{u}}$

\section{MACRO Collaboration}

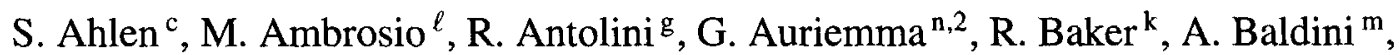
G.C. Barbarino ${ }^{\ell}$, B.C. Barish ${ }^{d}$, G. Battistoni ${ }^{\text {ft, }, 11}{ }^{\text {, R. Bellotti }}{ }^{a}$, C. Bemporad ${ }^{m}$, P. Bernardini ${ }^{j}$,

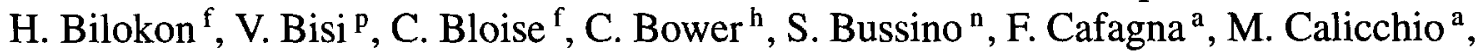

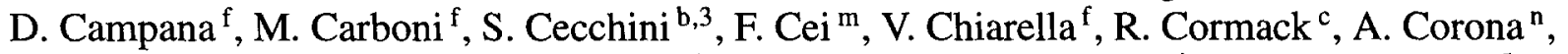
S. Coutu ${ }^{k}$, G. DeCataldo ${ }^{a}$, H. Dekhissi ${ }^{\text {b,4 }}{ }^{\text {, C. DeMarzo }}{ }^{\text {a }}$, I. De Mitri ${ }^{i}$, M. De Vincenzi ${ }^{n, 5}$, A. Di Credico ${ }^{g}$, E. Diehl ${ }^{k}$, O. Erriquez ${ }^{a}$, C. Favuzzi ${ }^{a}$, D. Ficenec ${ }^{c, 6}$, C. Forti ${ }^{f}$, P. Fusco ${ }^{a}$,

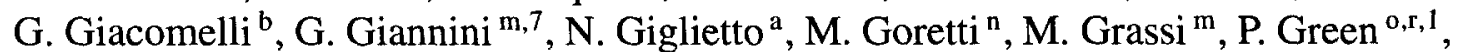

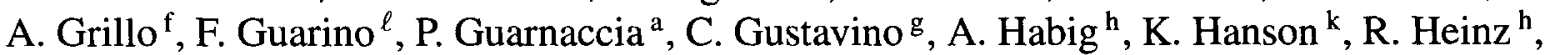
J.T. Hong ${ }^{d, c, 1}$, E. Iarocci ${ }^{\text {f, } 8}$, E. Katsavounidis ${ }^{d}$, E. Kearns ${ }^{c}$, S. Klein ${ }^{c, 9}$, S. Kyriazopoulou ${ }^{d}$, E. Lamanna ${ }^{n}$, C. Lane ${ }^{\mathrm{e}}$, D.S. Levin ${ }^{\mathrm{k}}$, P. Lipari ${ }^{\mathrm{n}}$, G. Liu ${ }^{\mathrm{d}}$, R. Liu ${ }^{\mathrm{d}}$, M.J. Longo $^{\mathrm{k}}$, Y. Lu ${ }^{\circ}$, G. Ludlam ${ }^{c}$, G. Mancarella ${ }^{j}$, G. Mandrioli ${ }^{b}$, A. Margiotta-Neri ${ }^{b}$, A. Marin $^{c}$, A. Marini ${ }^{f}$, D. Martello ${ }^{\mathrm{j}, \mathrm{q}, 1}$, A. Marzari Chiesa ${ }^{\mathrm{p}}$, P. Matteuzzi ${ }^{\mathrm{b}}$, M.N. Mazziotta ${ }^{\mathrm{a}}$, D.G. Michael ${ }^{\mathrm{d}}$, S. Mikheyev ${ }^{\mathrm{g}, 10}$, L. Miller $^{\mathrm{h}}$, M. Mittelbrun ${ }^{\mathrm{e}}$, P. Monacelli $^{\mathrm{i}}$, T. Montaruli $^{\mathrm{a}}$, M. Monteno $^{\mathrm{p}}$,

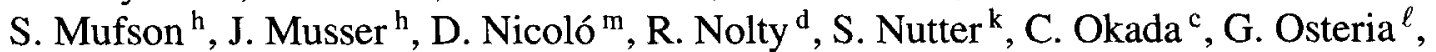

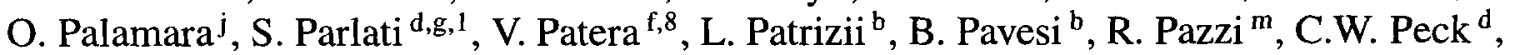

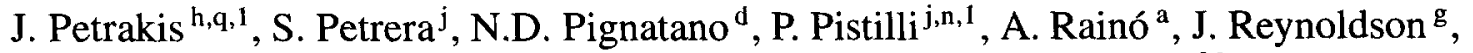
F. Ronga ${ }^{f}$, G. Sanzani ${ }^{b}$, A. Sanzgiri ${ }^{\circ}$, F. Sartogo ${ }^{n}$, C. Satriano ${ }^{n, 2}$, L. Satta $^{\text {f,8 }}{ }^{\text {, E. Scapparone }}{ }^{\text {b }}$, K. Scholberg ${ }^{\text {d }}$, A. Sciubba ${ }^{\text {f, }}$, , P. Serra Lugaresi ${ }^{b}$, M. Severi ${ }^{n}$, M. Sitta ${ }^{p}$, P. Spinelli ${ }^{a}$,

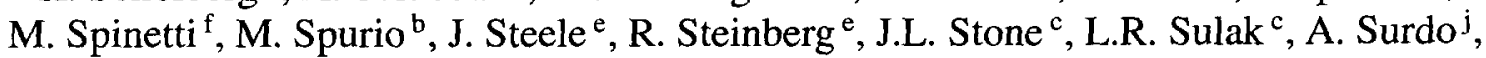
G. Tarlék ${ }^{k}$, V. Togo ${ }^{b}$, V. Valente ${ }^{\mathrm{f}}$, C.W. Walter ${ }^{\mathrm{d}}$, R. Webb ${ }^{\mathrm{o}}$, W. Worstell ${ }^{\mathrm{c}}$

a Dipartimento di Fisica dell'Università di Bari and INFN, Bari, 70126, Italy 
${ }^{b}$ Dipartimento di Fisica dell'Università di Bologna and INFN, Bologna, 40126, Italy

c Physics Department, Boston University, Boston, MA 02215, USA

d California Institute of Technology, Pasadena, CA 91125, USA

' Department of Physics, Drexel University, Philadelphia, PA 19104, USA

f Laboratori Nazionali di Frascati dell'INFN, Frascati (Roma), 00044, Italy

g Laboratori Nazionali del Gran Sasso dell'INFN, Assergi (L'Aquila), 67010, Italy

${ }^{\mathrm{h}}$ Depts. of Physics and of Astronomy, Indiana University, Bloomington, IN 47405, USA

'Dipartimento di Fisica dell'Università dell'Aquila and INFN, L'Aquila, 67100, Italy

i Dipartimento di Fisica dell'Università di Lecce and INFN, Lecce, 73100, Italy

${ }^{\mathrm{k}}$ Department of Physics, University of Michigan, Ann Arbor. MI 48109, USA

${ }^{\ell}$ Dipartimento di Fisica dell'Università di Napoli and INFN, Napoli, 80125, Italy

m Dipartimento di Fisica dell'Università di Pisa and INFN, Pisa, 56010, Italy

"Dipartimento di Fisica dell'Università di Roma and INFN, Roma, 00185, Italy

- Physics Department, Texas A\&M University, College Station, TX 77843, USA

P Dipartimento di Fisica dell'Università di Torino and INFN, Torino, 10125, Italy

q Bartol Research Institute, University of Delaware, Newark, DE 19716, USA

r Sandia National Laboratory, Albuquerque, NM 87185, USA

s Istituto di Cosmo-Geofisica del CNR. Torino, 10133, Italy

' INFN Sezione di Milano, 20133, Italy

"Istituto di Fisica dell'Università di Torino and INFN, Torino, 10125, Italy

${ }^{v}$ Instituto de Fisica, Universidade Estadual de Campinas, Campinas, 13081 SP, Brazil

Received 2 August 1994

Editor: K. Winter

\begin{abstract}
A study of the primary cosmic ray composition in the energy range $5 \cdot 10^{14}-5 \cdot 10^{15} \mathrm{eV}$ is performed through the analysis of the deep underground muons and of the e.m. component of Extensive Air Showers detected in coincidence by the MACRO and EAS-TOP experiments at the Gran Sasso Laboratory. We conclude that: a) pure proton and Iron primary beams are excluded by the experimental data; b) a fraction of nuclei heavier than Helium is necessary to explain the experimental data both below and above the knee; c) a mixed composition, obtained a priori from extrapolations of the spectra directly measured below $100 \mathrm{TeV}$, yields a reasonable account of the observations up to the knee of the primary spectrum.
\end{abstract}

1 Current address

2 Also Università della Basilicata, Potenza, 85100, Italy

3 Also Istituto TESRE/CNR, Bologna, Italy

4 Also at Faculty of Science, University Mohamed I, Oujda, Morocco

5 Also at Università di Camerino, Camerino, Italy

${ }^{6}$ Now at Physics Department, Washington University, St Louis, MO 63130, USA

7 Also Università di Trieste and INFN, Trieste, 34100, Italy

8 Also Dipartimento di Energetica, Università di Roma, Roma, 00185 , Italy

9 Now at Department of Physics, University of California, Santa Cruz, CA 95064, USA

${ }^{10}$ AIso at Institute for Nuclear Research, Russian Academy of Sciencee, Moscow, Russia

"Mail address: INFN Sezione di Milano, via Celoria 16, 20133 Milano, Italy; tel.: +39-2-239 2307, fax: +39-2-239 23624; email: battistoni@vaxmi.mi.infn.it
The measurement of the primary composition at high energies $\left(\geq 10^{14} \mathrm{eV}\right)$ and of its possible variations around the steepening of the primary spectrum (the "knee", at about $2 \cdot 10^{15} \mathrm{eV}$ ), is one of the main experimental problems in cosmic ray physics. Due to the rather low fluxes, measurements must be indirect, i.e. through the study of the EAS components. Measurements are then sensitive not only to the primary spectrum and composition, but also to the interaction properties. Moreover, in the energy region of interest, no direct hadron-nucleus and nucleus-nucleus accelerator measurements are available, and the lower energy hadronic interaction data have to be extrapolated. Experiments should therefore guarantee the possibility of testing and comparing the main features of different interaction models. 
The shower size $\left(N_{e}\right)$, and the TeV muon content $\left(N_{\mu}\right)$ are two quantities relevant to this investigation. Their simultaneous measurement can provide information on the mass composition as a function of the primary energy. $N_{e}-N_{\mu}$ data are recorded at the Gran Sasso Laboratory by EAS-TOP [1] and MACRO [2], operating in coincidence mainly in the primary energy range $10^{14}-10^{16} \mathrm{eV}$. First results on their combined operation and resolutions have been published in [3], and a first analysis in terms of primary composition has been presented in [4].

In this paper we investigate the compatibility of our experimental data with:

a) extreme compositions, made of pure nuclear species $(\mathrm{H}, \mathrm{He}, \mathrm{Fe})$ that are defined both below and above the knee of the spectrum;

b) the " $\Sigma$ model", a composition resulting from an extrapolation of the spectra obtained through direct observations at lower energies, defined only below the knee.

We also discuss some effects of the possible systematics arising from the adopted interaction model and cascade simulation.

A comparative discussion of the present data with other experimental results and composition models is not within the aim of this paper and will be presented elsewhere.

EAS-TOP, at Campo Imperatore (2000 m a.s.1.), is an apparatus tailored for the detection of several different EAS components [1]: the e.m. component, hadrons, muons, Cherenkov light, and radio emission. The e.m. detector of EAS-TOP, used for the measurement of shower size $N_{e}$, is an array of 35 modules of scintillator, each of area $10 \mathrm{~m}^{2}$, distributed over $\sim 10^{5} \mathrm{~m}^{2}$. In this work we consider only internal trigger events, i.e. events in which the maximum number of particles is detected by an inner module, and at least seven counters fired. For these events, primary arrival direction, core location and shower size are reconstructed with accuracies $\Delta \theta=0.5^{\circ}, \Delta r \leq 10 \mathrm{~m}$, and $\Delta N_{e} / N_{e} \leq 20 \%$ for $N_{e} \geq 10^{5}$. A detailed discussion of the EAS-TOP e.m. reconstruction techniques and accuracies is given in Ref. [5]. To be consistent with the simulations, in the present paper the particle densities, from which $N_{e}$ is derived, are measured in units of the energy released in the scintillators divided by $9.8 \mathrm{MeV}$, which is the average energy lost by a minimum ionizing particle at a $33.5^{\circ}$ zenith an- gle, i.e. the average angle along which the two experiments are aligned. Systematic effects have been explored by comparing different theoretical expressions of the electron lateral distribution function (still compatible with the experimental measurements), and are below the 5\% level.

MACRO, in the underground Gran Sasso Laboratory at $963.45 \mathrm{~m}$ a.s.l., $3100 \mathrm{~m}$ w.e. of minimum rock overburden, is a large area multi-purpose apparatus designed to detect penetrating cosmic radiation. The lower part of the MACRO detector has dimensions $76.6 \times 12 \times 4.8 \mathrm{~m}^{3}$. It has a modular structure whose basic unit is a supermodule of size $12.6 \times 12 \times 4.8$ $\mathrm{m}^{3}$, equipped with planes of limited streamer tubes with two-dimensional (wire and strip) digital readout, track-etch plastic modules and liquid scintillator counters [2]. In this work we consider only muon tracks, which are required to have at least 4 aligned hits in both views of the horizontal streamer tube planes. The angular resolution for muon tracks is less than one degree, dominated by multiple scattering in the rock. Space point accuracy is of the order of 1 $\mathrm{cm}$, and track resolution is at the level of $5 \mathrm{~cm}$. A discussion of the error in the measurement of muon multiplicity is given in Ref. [7]. For this analysis all events have been visually scanned, reducing the typical systematic uncertainty in $N_{\mu}$ reconstruction to \pm 1 at the highest observed multiplicities.

The two experiments are separated by a thickness of rock ranging from 1100 up to 1300 meters, depending on the angle. The corresponding minimum energy for a muon to reach the depth of MACRO ranges from $\sim 1.3$ to $\sim 1.8 \mathrm{TeV}$. Event coincidence is established off-line, using the absolute time given by atomic clocks with an accuracy better than $1 \mu$ s. Three runs have been performed with the detectors operating in coincidence. In this paper we present the data recorded during winter 1992-1993 with the full EAS-TOP e.m. array, and the complete lower part of MACRO. The total live time is 87.5 days. By imposing the reconstruction criteria described above, in this period we have recorded 1821 events within $\pm 2 \mu$ s of the coincidence peak, the expected accidental contamination being $2.8 \%$.

Experimental data are compared with the results of a full simulation of the primary interaction, the development of the e.m. and hadronic shower in the atmosphere, the muon propagation in the rock, and the response of both detectors. The physics generator is 
contained in the HEMAS Monte Carlo code [8]. It is based on the UA5 multi-cluster parameterization of minimum bias events in $p-p$ and $p-\bar{p}$ colliders, up to $\sqrt{s}=900 \mathrm{GeV}$. Extrapolation to higher energies is made in the context of the inelastic "log $s$ physics". Nuclear target effects are also included according to experimental data up to an energy of $400 \mathrm{GeV}$ in the laboratory frame. The nuclear projectiles are considered in the context of the superposition model [9]. The hadronic shower and the muon component in the atmosphere are followed down to an energy cut of $1 \mathrm{TeV}$. The HEMAS code also provides three-dimensional muon propagation in standard rock. The e.m. component of the shower at the EAS-TOP depth, as generated by gamma-rays and electrons of any energy, and by the interactions of charged hadrons with an energy below the quoted energy cut, is obtained from the parameterization of the results of a detailed simulation of showers using GEANT [10], FLUKA [11], and EGS [12] codes with an energy cut of $1 \mathrm{MeV}$. This interaction model has also been used by the MACRO experiment in the study of the separation of underground muons [6], and of cosmic ray composition through the analysis of the multiplicity distribution of the muon events undeground [7]; it has also been used for the study of the e.m. size spectrum as measured by the EAS-TOP experiment alone [13].

We have adopted the following simulation strategy. In the event generator, after sampling mass, energy and direction of the primary particle, we calculate, event by event, the shower size at surface level and the kinematic variables of muons survived underground. For each event the impact position of shower axis is randomized over an area much larger than that of underground experiment, while the e.m. shower front is accordingly moved over the surface array. If at least a segment of track enters the acceptance area of MACRO, a detailed simulation of detector and trigger is started for both surface and underground apparata. If at this simulation stage the trigger conditions of both detectors are fulfilled, the event is stored as a coincidence event. The same data structure of real events is used, so that simulated events are processed by the same reconstruction codes employed for real data. The reconstructed e.m. size at EAS-TOP level and the reconstructed muons tracks in MACRO are then kept for the analysis. We have simulated a statistical sample corresponding to 2.8 times the experimental one.
In order to summarize some results of our Monte Carlo event generator, and to define the relevant energy range of the coincidence experiment, we report here a parameterization of the conversion between average size and primary energy:

$N_{e}\left(E_{0}, A\right)=\alpha(A) \cdot E_{0}^{\beta(A)}$

where $\alpha(A)=99.8 \cdot A^{-0.33}$ and $\beta(A)=1.15 \cdot A^{0.012}$, and $E_{0}$ is the energy/nucleus in TeV. The analogous parameterization for the number of muons underground (for an infinite area detector) are given in Ref. [8]. However, none of these parameterizations is used in the present analysis.

As mentioned in the introduction, for any given input spectrum and composition, the main source of systematic uncertainties in such analyses is due to the insufficiencies of the interaction model.

MACRO has tested the validity of some fundamental features of the interaction model, through the anal$y$ sis of the distribution of the underground muon separation [6], obtaining a good consistency. This test is also essential for the muon multiplicity analysis in a finite size detector.

We also performed a preliminary comparative check of the systematics associated to the interaction model using the SIBYLL code [14], which is based on the Dual Parton Model [15] with the inclusion of minijets. The results indicate that, in the energy range relevant for the combined experiments, the average e.m. size is practically unchanged with respect to the HEMAS results $(<0.3 \%)$, while the average muon multiplicity underground, $\left\langle N_{\mu}\right\rangle$, (for an infinite area detector) varies at most by $10 \%$. This was already anticipated in the review of Ref. [16], where other models were considered. Systematic effects are also due to the uncertainties in the rock composition, density and thickness. By including all of them in a $2 \%$ error in the thickness, we obtain a systematic uncertainty of $8 \%$ in the average muon multiplicity for protons at $100 \mathrm{TeV}$, decreasing to $1.6 \%$ at $1000 \mathrm{TeV}$. We have additionally verified that a variation of $10 \%$ in the simulated $\left\langle N_{\mu}\right\rangle$ for an infinite area detector, results only in a $3 \%$ difference in $\left\langle N_{\mu}^{\text {det }}\right\rangle$ in our set-up. It has to be noticed that the amplitude of the discussed systematic effects (uncertainties in the survival probabilities of muons at a given rock depth, relative importance of particle production in the frag- 
Table 1

Integral flux at $100 \mathrm{TeV}\left(\mathrm{m}^{-2} \cdot \mathrm{sr}^{-1} \cdot \mathrm{s}^{-1}\right)$ and spectral indices for the pure mass spectra.

\begin{tabular}{lllll}
\hline Mass group & Integral flux & $\gamma^{(1)}-1$ & $\mathrm{E}_{\mathrm{knce}}(\mathrm{GeV})$ & $\gamma^{(2)}-1$ \\
\hline $\mathrm{p}$ & $3.16 \cdot 10^{-5}$ & 1.88 & $1.83 \cdot 10^{6}$ & 2.19 \\
$\mathrm{He}$ & $6.19 \cdot 10^{-5}$ & 1.92 & $2.39 \cdot 10^{6}$ & 2.24 \\
$\mathrm{Fe}$ & $1.87 \cdot 10^{-4}$ & 1.99 & $3.85 \cdot 10^{6}$ & 2.32 \\
\hline
\end{tabular}

mentation region, calculation of size value, of its fluctuations, and its measurements) are known to decrease with primary energy. This conclusion is reinforced by our simulation tests. However, these factors are still subject of further theoretical and experimental investigation.

In this analysis, we required that any tested input spectrum and composition should reproduce the e.m. size spectrum as measured by the surface experiment alone [13]. The quoted experimental result of EAS-TOP shows how the size spectrum steepens, with a variation in the spectral index $\Delta \gamma=0.5 \pm 0.1$ ("knee"), at $N_{e}=10^{5.75}$, for the present definition of $N_{e}$, and at the average zenith angle of the coincidence experiment.

Two kinds of primary beams were tested:

1) A set of pure nuclear species with energy spectra constructed to fit the size spectrum measured by EAS-TOP alone [13]. The usual approximation of two power law energy spectra, meeting at a mass dependent knee energy has been used: $A_{i}^{(1,2)} \cdot E^{-\gamma_{i}^{(1,2)}} \cdot d E$, where the superscripts 1 and 2 refer to the energy range below and above the knee, respectively. The parameters of the integral spectra for the pure mass species are given in Table 1 .

2) An a priori constructed mixed composition obtained by the combination of spectra fitting direct measurements from balloons and satellites at 1-100 $\mathrm{TeV}$, extrapolated up to the knee region. The proton and Helium spectra are derived from the 1991 JACEE data [17], the heavier mass groups' spectra from the CRN data [18]. This set of spectra, that we call $\Sigma$ model, was found to reproduce the EAS-TOP measured size spectrum [13], within the experimental uncertainties, in the size range $N_{e} \sim 10^{5.25}-10^{5.75}$, i.e. just below the knee. The integral flux at $100 \mathrm{TeV}$ and the relative spectral indices, with fitting errors, are given in Table 2 . The $\langle A\rangle$ value predicted by the
Table 2

Integral flux at $100 \mathrm{TeV}\left(\mathrm{m}^{-2} \cdot \mathrm{sr}^{-1} \cdot \mathrm{s}^{-1}\right)$ and spectral indices of the nuclear components of the $\Sigma$ Model.

\begin{tabular}{lll}
\hline Mass group & Integral Flux & $\gamma-1$ \\
\hline $\mathrm{p}$ & $(1.33 \pm 0.31) \cdot 10^{-5}$ & $1.86 \pm 0.07$ \\
$\mathrm{He}$ & $(2.28 \pm 0.44) \cdot 10^{-5}$ & $1.72 \pm 0.09$ \\
$\mathrm{CNO}$ & $(7.09 \pm 0.48) \cdot 10^{-6}$ & $1.67 \pm 0.06$ \\
$\mathrm{Mg}-\mathrm{Si}$ & $(2.48 \pm 0.15) \cdot 10^{-6}$ & $1.82 \pm 0.11$ \\
$\mathrm{Fe}$ & $(6.94 \pm 0.63) \cdot 10^{-6}$ & $1.60 \pm 0.11$ \\
\hline
\end{tabular}

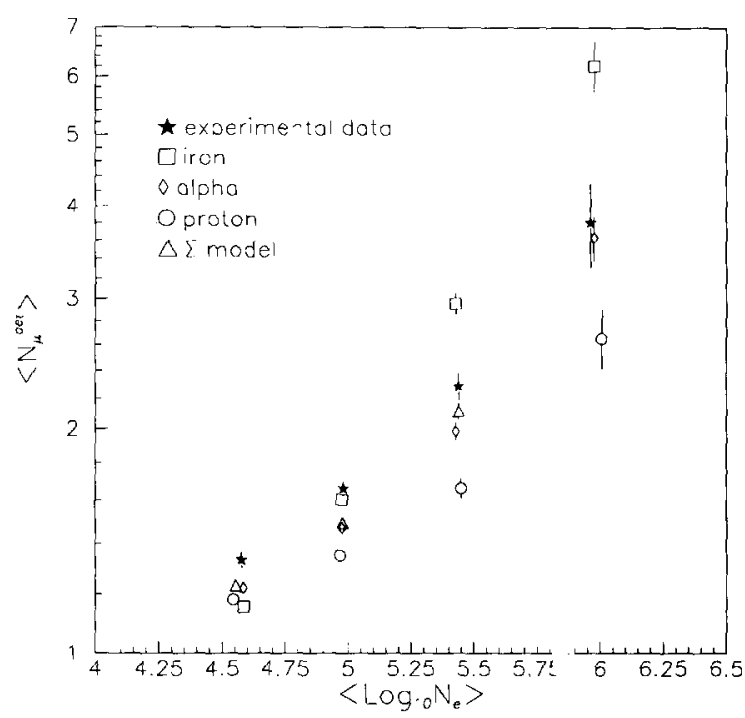

Fig. 1. Correlation between $\left\langle N_{\mu}^{\text {det }}\right\rangle$ and $\left\langle\log _{10} \mathrm{~N}_{e}\right\rangle$. Only statistical errors are shown, while systematic uncertainties are discussed in the text.

model (using as weight the differential spectrum in energy/nucleus) smoothly increases with energy, and reaches the value of 14.4 at $1000 \mathrm{TeV}$. No extension is made of these extrapolations above the knee.

The most significant physical information is obtained through the $N_{e}-N_{\mu}$ correlation, which depends primarily on the cosmic ray composition and is largely independent of the energy spectrum. Fig. 1 shows the relationship between the average detected muon multiplicity $<N_{\mu}^{\text {det }}>$ and $\log _{10} N_{e}$. The events are divided into four size intervals, the knee of the spectrum (at $N_{e} \simeq 10^{5.75}$ ) being located at the border between the last two bins. From this plot we derive the following conclusions:

1) The method is essentially sensitive to the primary composition in the last two size bins, where not only 
the different mass groups can be better separated, but also the known systematic uncertainties, as discussed above, are expected to be less relevant than in the lower energy region. For these reasons it is difficult to draw any conclusion from the first two bins.

2) In the range $10^{5.25}<N_{e}<10^{5.75}$, i.e. below the knee, the experimental point is:

a) inconsistent with the predictions obtained by assuming a primary beam composed of only protons or Fe nuclei, statistical inconsistencies being at the level of $5 \sigma$, and remaining at a level of $3 \sigma$ even when including the maximum possible systematics.

b) consistent with an intermediate mass composition $(A \geq 4)$, and in particular with the prediction of the $\Sigma$ model.

3) At $\mathrm{N}_{e}>10^{5.75}$, i.e. above the knee, where only pure compositions are considered, the remaining sample of 72 measured coincident events still appears to be incompatible with the hypothesis of pure $\mathrm{p}$ or Fe compositions, with statistical inconsistencies (limited by the size of the sample) being in this case at the level of $2 \sigma$. An intermediate mass composition is again the best interpretation of the data.

Further information can be inferred by examining the muon multiplicity distribution in the range $10^{5.25}<$ $N_{e}<10^{5.75}$ shown in Fig. 2. Unlike Fig. 1, such a distribution shows absolute rates of events. For pure compositions the errors in these rates are determined by the maximum quoted systematic uncertainty in the normalization of size spectrum as measured by EASTOP alone. In the case of $\Sigma$ model, the errors are determined by the uncertainties in the extrapolated fluxes, that, considering the maximum possible error, can be as large as $20 \%$ for a single nuclear species ( see Table 2).

In order to take into account the maximum systematic uncertainties in the statistical estimates, we adopt the following conservative approach. The overall effect of systematics can be projected, for a given e.m. size bin, into an uncertainty on the simulated muon multiplicity. In practice, the maximum error in our analysis corresponds to one unit in the muon multiplicity. Therefore we compare the number of observed events above a given $N_{\mu}^{\text {det }}$ in the experimental live time $T_{\exp }$, with the calculated number of events with multiplicity above $N_{\mu}^{\text {det }}-1$, as obtained in the simulation live time $T_{\text {sim }}$.

We observe:

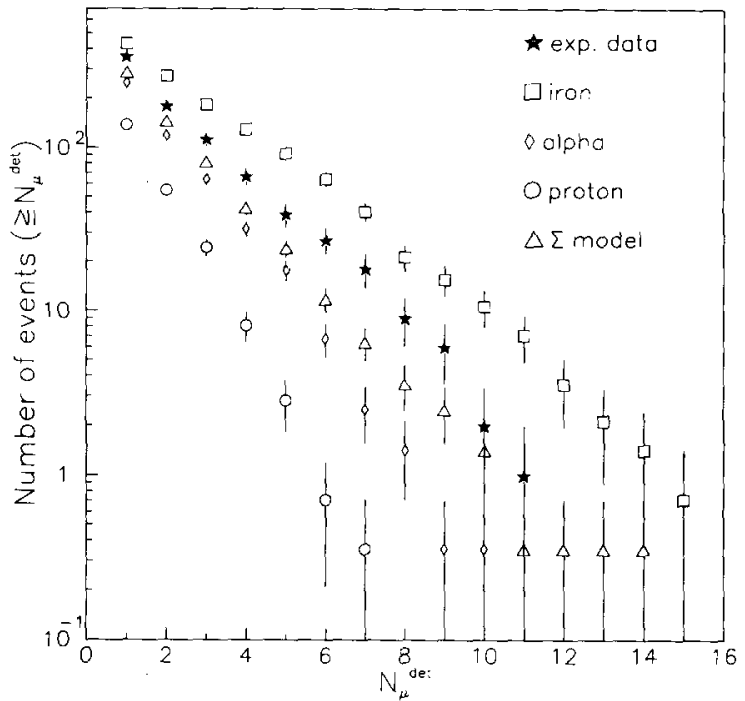

Fig. 2. Measured and expected integral distribution of detected muon multiplicities, for $5.25 \leq \log _{10} N_{e} \leq 5.75$. As explained in the text, the maximum systematic uncertainty is equivalent to one unit in the simulated $N_{\mu}^{\mathrm{det}}$.

a) the measured rate of high muon multiplicities cannot be explained by pure Helium primaries. In fact, according to the above described procedure, we observe 19 events with $N_{\mu}^{\text {det }} \geq 7$ (experimental live time $T_{\exp }=$ 87.5 days) against 18 expected events with $N_{\mu}^{\text {det }} \geq 6$ (simulation live time $T_{\operatorname{sim}}=245$ days). When the different live times are allowed for, the probability that the observed and predicted number of events are sampled from the same parent distribution is $2.8 \cdot 10^{-3}$. This shows that, although pure Helium primaries are still compatible with the $\left\langle N_{\mu}^{\text {det }}>-\log _{10} N_{e}\right.$ plot of Fig. 1, they cannot explain the tail of the $N_{\mu}^{\text {det }}$ distributions. A fraction of higher mass primaries, and therefore a mixed composition, is required.

b) The $\Sigma$ model also seems to predict a lower tail at high multiplicities than the experimental data; however, if the total systematic uncertainty in the extrapolated flux is allowed to be just as large as $30 \%$, the need to increase the amount of heavy primaries in the model is not compelling. The same uncertainty on the flux can explain the event rate, in fact the total number of recorded events in the considered size bin is 359 , while the expected one from the $\Sigma$ model is 288 (i.e. within 25\%).

In Fig. 3 we show the similar multiplicity distribu- 


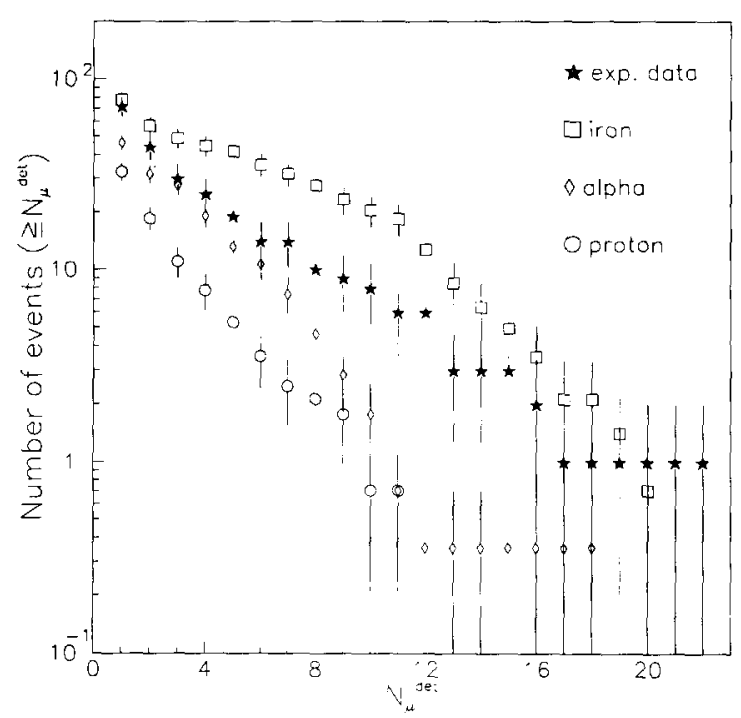

Fig. 3. Measured and expected integral distribution of detected muon multiplicities, for $\log _{10} N_{e} \geq 5.75$. As explained in the text, the maximum systematic uncertainty is equivalent to one unit in the simulated $N_{\mu}^{\text {det }}$.

tion for the events above the knee, where of course the $\Sigma$ model is not present. Again a pure Helium beam, although consistent with the data of Fig. 1, cannot explain the tail of the high muon multiplicities. We observe 6 events with $N_{\mu}^{\text {det }} \geq 12\left(T_{\exp }=87.5\right.$ days $)$ against 2 events expected with $N_{\mu}^{\text {det }} \geq 11\left(T_{\text {sim }}=245\right.$ days). The probability that this excess is due to a random fluctuation is $5.5 \cdot 10^{-3}$. This gives evidence for the presence of $A \geq 4$ nuclei in cosmic rays above the knee, and of a mixed composition also in this energy range.

We conclude that the coincident EAS-TOP and MACRO data, in the energy range $5 \cdot 10^{14}-5 \cdot 10^{15}$ $\mathrm{eV}$, are reasonably explained, below the knee, by the extrapolation of the direct measurements performed at lower energies. Unless substancial changes in the hadronic interaction process are invoked, the presence of elements heavier than Helium is necessary in the whole considered energy range. Furthermore, these data do not favour the hypothesis of a dramatic change towards pure extreme compositions around the knee of the cosmic ray spectrum.
We gratefully acknowledge the support of the director and staff of the Gran Sasso National Laboratory, and the invaluable assistance of the technical staff of the institutions participating in the experiments. This work was supported in part by the Italian National Institute for Nuclear Physics (INFN), the Italian National Research Council (CNR), the US Department of Energy, and the US National Science Foundation.

\section{References}

[1] EAS-TOP Collaboration (M. Aglietta et al.), Nucl, Instr. \& Meth. A 277 (1989) 23.

[2] MACRO Collaboration (S.P. Ahlen et al.), Nucl. Instr. \& Meth. A 324 (1993) 337.

[3] MACRO and EAS-TOP Collaborations (R. Bellotti et al.), Phys. Rev. D 42 (1990) 1396.

[4] MACRO and EAS-TOP Collaborations, Proc. 22nd I.C.R. C., Dublin, 2 (1991) 61; Proc. 23rd I.C.R.C., Calgary, 2 (1993) 89.

[5] EAS-TOP Collaboration (M. Aglietta et al.), Nucl. Instr. \& Meth. A 336 (1993) 310.

[6] MACRO Collaboration (S.P. Ahlen et al.), Phys. Rev. D 46 (1992) 4836; Proc. 23rd I.C.R.C., Calgary, 2 (1993) 93.

[7] MACRO Collaboration (S.P. Ahlen et al.), Phys. Rev. D 46 (1992) 895; Proc. 23rd I.C.R.C., Calgary, 2 (1993) 97.

[8] C. Forti et al., Phys. Rev. D 42 (1990) 3668.

[9] T.K. Gaisser et al., Phys. Rev. D 25 (1982) 2341.

[10] R. Brun et al., report CERN DD/EE/84-1 (1984).

[11] A. Fassó et al., "FLUKA: present status and future developments", IV Int. Conf. on Calorimetry in High Energy Physics, La Biodola (Is. d'Elba), Italy, 1993, Proc., in press.

[12] W.R. Nelson, H. Hirayama and D.W.O. Rogers, report SLAC-265 (1985).

[13] EAS-TOP Collaboration, Proc. 23rd I.C.R.C., Calgary, 4 (1993) 247.

[14] P. Lipari et al, Proc. 22nd I.C.R.C., Dublin, 4 (1991) 197; R.S. Fletcher et al." "SIBYLL: An event generator fo simulation of high energy cosmic ray cascades", preprint of Bartol Research Institute (1994) BA-94/01, submitted to Phys. Rev. D.

[15] See for instance A. Capella et al., Phys. Rev. Lett. 58 (1987) 2015 , and references therein.

[16] T.K. Gaisser, Proc. Vulcano Workshop 1992 "Frontier Objects in Astrophysics and Particle Physics", 40 (1993) 433; see also Ref. [14].

[17] JACEE Collaboration (T.H. Burnett et al.), Ap.J., 349 (1990) L25;

K. Asakimori, Proc. 22nd I.C.R.C., Dublin, 2 (1991) 57.

[18] CRN Collaboration, D. Muller et al., Ap. J. 374 (1991) 356. 